J Hosp Infect. 2019 January ; 101(1): 69-75. doi:10.1016/j.jhin.2018.07.018.

\title{
The Local Hospital Milieu and Healthcare-Associated VRE Acquisition
}

\author{
Margaret J. Zhou, MD1, Jianhua Li, PhD², Hojjat Salmasian, MD, MPH, PhD ${ }^{3}$, Philip \\ Zachariah, MD, MS ${ }^{4}$, Yu-Xiao Yang, MD $^{5}$, and Daniel E. Freedberg, MD, MS ${ }^{6}$ \\ ${ }^{1}$ Department of Medicine, Columbia University Medical Center, New York, New York, USA \\ 2Biomedical Informatics, Department of Medicine, Columbia University Medical Center, New York, \\ New York, USA \\ ${ }^{3}$ Data Science and Evaluation, Brigham and Women's Hospital, Boston, Massachusetts, USA \\ ${ }^{4}$ Department of Pediatrics, Columbia University Medical Center, New York, New York, USA \\ ${ }^{5}$ Division of Gastroenterology, Perelman School of Medicine, University of Pennsylvania, \\ Philadelphia, Pennsylvania, USA \\ ${ }^{6}$ Division of Digestive and Liver Diseases, Columbia University Medical Center, New York, New \\ York, USA
}

\section{Abstract}

Background-Vancomycin-resistant Enterococcus (VRE) causes 4\% of all healthcare-associated infections in the United States. The process by which the local hospital milieu contributes to VRE acquisition is not fully understood.

\begin{abstract}
Aim-To determine the importance of specific factors within the local hospital environment for healthcare-associated VRE acquisition.

Methods-This retrospective cohort study included patients admitted to 6 intensive care units (ICUs) at an academic medical center from January 2012 to December 2016 with negative rectal VRE cultures on admission. VRE acquisition was defined as a positive surveillance swab performed at any time after the initial negative swab during the index hospitalization. The exposures of interest were VRE colonization pressure, VRE importation pressure, and use of vancomycin. Multivariable Cox proportional hazards modeling was performed, with patients followed until VRE acquisition, death, or for up to 30 days.
\end{abstract}

Findings-Of 8,485 patients who were initially VRE-negative, 161 patients acquired VRE. On univariate analysis, patients with VRE acquisition were more likely to have received vancomycin, have had a neighboring patient who received vancomycin, have high VRE importation pressure, or have high VRE colonization pressure. On multivariable analysis, only high VRE colonization pressure was an independent predictor of VRE acquisition (adjusted hazards ratio [aHR] 1.79, $95 \%$ confidence interval $[\mathrm{CI}] 1.19-2.70)$.

Contact information for corresponding author: Daniel E. Freedberg, Columbia University Medical Center, 630 West $168^{\text {th }}$ Street, New York, NY 10032, Telephone: (212) 305-1021, Fax: 212-305-5576, def2004@ cumc.columbia.edu. 
Conclusion-VRE colonization pressure was the most important risk factor for healthcareassociated VRE acquisition, regardless of VRE importation pressure. Interventions seeking to reduce VRE acquisition should focus on minimizing transmission between patients with known VRE and the local hospital environment.

\section{INTRODUCTION}

Vancomycin-resistant Enterococcus (VRE) causes 4\% of all healthcare-associated infections (HAIs) in the United States, making it the second most common multidrug-resistant HAI behind MRSA. ${ }^{1}$ Case fatality rates for VRE infections range from $17 \%$ to $50 \%$ depending on the site of infection. ${ }^{2,3}$ In hospitalized patients, gastrointestinal VRE colonization is common, especially in the intensive care unit (ICU) where rates of VRE colonization on admission range from $4 \%$ to $30 \% .^{4-7}$ Although the majority of patients with VRE colonization never develop subsequent VRE infections, VRE colonization appears to be a marker for loss of normal gastrointestinal colonization resistance against pathogens. ${ }^{5}$ In the ICU, VRE-colonized patients have longer ICU stays, increased hospital costs, and increased mortality compared to non-colonized patients. ${ }^{8}$

VRE persists on hard surfaces for weeks to months. ${ }^{9}$ Cultures of ICU room surfaces remain positive for VRE in $10 \%$ to $50 \%$ of rooms after cleaning, suggesting that VRE outbreaks may be attributed to fomites and transmission via healthcare workers. ${ }^{10,11}$ Standard infection control approaches to VRE in high-risk settings include surveillance swabs, vancomycin stewardship, environmental cleaning, and contact precautions, although the relative effectiveness of these measures in reducing transmission is unclear. ${ }^{12-14}$

The process by which the local hospital milieu contributes to VRE acquisition is not fully understood. Previous studies on VRE and other enteric organisms like Clostridium difficile have suggested that the rate at which the organism is imported into the institution, the rate of ward- or institution-level antibiotic use, and factors related to the prior bed occupant may all play a role in healthcare-associated acquisition. ${ }^{15-17}$ However, the relative contribution of these factors is far less certain. Elucidating the role of these local factors has the potential to improve understanding of VRE transmission and to target infection prevention and control resources towards the highest-yield prevention strategies.

The objective of this study was to better understand the relative importance of local factors that impact healthcare-associated VRE acquisition by examining risk factors related to the hospital milieu, specifically those related to circulating VRE (reflecting isolation measures), VRE burden prior to admission (reflecting environmental cleaning), and local use of vancomycin (reflecting antibiotic stewardship).

\section{METHODS}

\section{Study Population}

This was a retrospective cohort study including adults $\geq 18$ years old admitted to any of 6 ICUs affiliated with a large academic medical center from January 1, 2012 to December 31, 2016. If patients were admitted multiple times, only the first admission was included. 
Patients were excluded from the study if a VRE surveillance rectal swab was not performed within 24 hours of ICU admission, or if they had a positive VRE rectal swab upon admission. The study was approved by the institutional review board of Columbia University Medical Center with a waiver of informed consent.

\section{Healthcare-Associated VRE Acquisition}

The primary outcome was healthcare-associated VRE acquisition, defined as a positive subsequent VRE surveillance swab performed at any time after the initial negative surveillance swab during the same hospitalization. VRE surveillance cultures were collected within 1 hour of ICU admission by ICU nurses as flocked rectal swabs (Copan Diagnostics, Murrieta, CA, USA). ${ }^{18}$ These swabs were gathered with the patient in the left lateral decubitus position with the swab inserted deeply into the rectal canal and rotated 5 times. Swabs were transported in $1 \mathrm{~mL}$ of liquid Amies media via vacuum container tube for direct culture onto chromogenic differential media impregnated with $6 \mu \mathrm{g} / \mathrm{ml}$ of vancomycin (Remel, Hartford, CT, USA). Plates were incubated at $33-37^{\circ} \mathrm{C}$ under aerobic conditions for 24 hours and interpreted categorically based on the presence of VRE. VRE was classified as present if raised, smooth, small to medium-sized colonies appeared that were either dark blue or purple (E. faecium) or light blue (E. faecalis). VRE was classified as absent if there was no growth after 24 hours, or if colonies were present lacking these morphological features. Sub-culture to non-selective media for susceptibility testing was not performed. Standard contact precautions and cleaning policies were in place in all units, including use of sodium hypochlorite to clean all environmental surfaces and patient care equipment. Additional contact precautions (gown and gloves) were implemented in all units for patients with VRE positivity.

\section{The Local Hospital Milieu}

Two categories of risk factors were examined to characterize the local hospital milieu: 1) factors related to the local presence of VRE immediately preceding and during each patient's hospitalization, and 2) factors related to the local use of vancomycin. Variables to describe the former included VRE colonization in the prior bed occupant and VRE colonization in a neighboring patient located in a room immediately adjacent to the subject. For each at-risk patient, VRE colonization pressure was calculated to encapsulate the concept of circulating VRE in a manner similar to Dubberke et al., ${ }^{19}$ and was defined as the sum of the daily number of VRE-positive patients in the same ICU during the patient's ICU admission (sum VRE pressure) divided by the length of the patient's ICU admission (length of time at risk):

$$
\text { VRE colonization pressure }=\frac{\sum \text { Daily Exposure to VRE-positive patients (sum VRE pressure) }}{\text { Length of time at risk }}
$$

VRE importation pressure was calculated to encapsulate the concept of residual environmental VRE using a modification of the method described by Brown et al., ${ }^{16}$ and was defined as the number of patient-days of VRE-positivity during the 30 days prior to each patient's ICU admission divided by the total number of patient-days for all patients admitted during the prior 30 days: 
VRE importation pressure $=\frac{\sum \text { Number of patient-days of VRE-positivity in preceding } 30 \text { days }}{\sum \text { Number of all patient-days }}$

These variables were then organized into tertiles.

Factors related to the local use of vancomycin included receipt of vancomycin by the subject after the initial negative VRE surveillance swab, receipt of vancomycin by the prior bed occupant while in the relevant bed, receipt of vancomycin by a neighboring patient, and unitlevel vancomycin use, calculated in a similar manner as VRE colonization pressure (the sum patient-days of vancomycin exposure for simultaneous patients on the unit, divided by the patient's time at risk). Receipt of vancomycin included oral or intravenous vancomycin at any dose or duration.

\section{Covariates}

We examined multiple co-variables as potential risk factors for VRE acquisition including sex, age, race, season of hospital admission, specific ICU, and comorbidities as measured by a modified Charlson comorbidity index (CCI). ${ }^{20} \mathrm{We}$ also examined laboratory results obtained from blood tests performed at ICU admission including sodium, creatinine, albumin level, hematocrit, and white blood cell count; as well as ICU therapies including receipt of broad-spectrum antibiotics, immunosuppressants, acid suppression medication, whether surgery had been performed in the 24 hours prior to admission, mechanical ventilation, or dialysis (either continuous renal replacement therapy or intermittent hemodialysis). These therapies were classified categorically based on their presence or absence after the initial negative VRE swab. Cut-offs for laboratory variables were based on standard laboratory cut points or were derived from APACHE IV or Sepsis-3, which included sodium $<135$ or $>145 \mathrm{mEq} / \mathrm{L}$, creatinine $>2 \mathrm{mg} / \mathrm{dL}$, albumin $<2.5 \mathrm{~g} / \mathrm{dL}$, hematocrit $<35 \%$, and white blood cells $<4$ or $>12 \times 10^{9} .{ }^{21,} 22$ All data was extracted from the electronic medical record using automated queries. Broad-spectrum antibiotics were classified based on their anticipated impact on the GI microbiome, which was inferred from their relationship with risk for $C$. difficile infection. These included antibiotics with anaerobic and Gram-negative coverage, specifically $\beta$-lactam/ $\beta$-lactamase inhibitor combination antibiotics, carbapenems, cephalosporins, fluoroquinolones, monobactams, and clindamycin. ${ }^{23}$ Immunosuppressants included systemic steroids at a minimum daily dose of 5 milligrams of prednisone or equivalent, calcineurin inhibitors, anti-metabolites, anti-TNFa agents, or mycophenolic acid. ${ }^{24}$

\section{Statistical Approach}

The final multivariable analysis was constructed using a Cox proportional hazards model with patients followed from the time of ICU admission until death, VRE acquisition, or for a maximum of 30 days. The proportional hazards assumption was verified by visual inspection of time-to-event data and by testing for a non-zero slope in the Schoenfeld residuals, and the log-rank test was used to assess for equality between survivor functions. ${ }^{25}$ To build the final model, variables were tested stepwise and included if they had a significant independent relationship with VRE acquisition or if they altered any of the $\beta$ - 
coefficients representing hospital milieu risk factors by at least $10 \%$. Sensitivity analyses were performed to test the robustness of our primary findings. These consisted of analyses within the final model restricted to specific subgroups of patients: (1) who had been re-tested for VRE colonization after the initial negative test (i.e., excluding patients who had only the initial VRE swab, and (2) who were admitted to the tertiary referral medical ICUs.

Additional sensitivity analyses were also conducted adjusting for the season of the year, and with VRE acquisition redefined as either a second positive surveillance swab or other culture newly positive for VRE. All analyses were performed using Stata statistical software (version 14, StataCorp) and were conducted as two-tailed p-values at the $a=.05$ level of significance.

\section{RESULTS}

\section{Population at Baseline}

A total of 8,485 patients were included, of which 161 (2\%) acquired VRE during the index hospital stay. All ICU rooms were single-occupancy. ICUs 1-4 were mixed medical-surgical ICUs where swabs were collected by provider discretion (32\% of all admissions swabbed). ICUs 5-6 were tertiary referral medical ICUs where admission VRE surveillance swabs were collected routinely ( $76 \%$ of all admissions swabbed). Compared to patients without VRE acquisition, those with VRE acquisition were more likely to be admitted to the tertiary care medical ICUs, have had recent surgery, required dialysis, required mechanical ventilation, received immunosuppressants, had elevated creatinine, or had hypoalbuminemia (Table I).

\section{Hospital Milieu Risk Factors}

In univariate analysis, patients with VRE acquisition were more likely to have received vancomycin or have a neighboring patient who received vancomycin (Table II). They were also more likely to have high VRE colonization pressure or high VRE importation pressure. Of these factors, high VRE colonization pressure was the most important covariate, with a VRE acquisition rate of $42 \%$ for those with high VRE colonization pressure versus $21 \%$ for those with low VRE colonization pressure (log-rank $\mathrm{p}<.01$, Figure 1).

\section{Multivariable Analysis}

The final multivariable analysis included the following risk factors: VRE colonization pressure, ICU location, Charlson comorbidity index, and elevated serum creatinine (Table III). When tested in this final model, other variables related to the hospital milieu no longer predicted VRE acquisition (Supplementary Table I). Similar results were obtained in the sensitivity analyses. There was no significant change after adjusting for season (aHR for high VRE colonization $1.81,95 \%$ CI 1.20 - 2.72), when the analysis was restricted to 1,131 patients who were rechecked for VRE during the index hospitalization (aHR 1.86, 95\% CI 1.03-3.36), or when the analysis was restricted to 3,305 patients admitted to the tertiary referral medical ICUs (aHR 1.90, 95\% CI 1.12 - 3.23). There was also no change when the analysis was conducted with VRE acquisition redefined as either a second positive surveillance swab or other culture newly positive for VRE $(n=181$, aHR $1.86,95 \%$ CI 1.03 $-3.36)$. 


\section{DISCUSSION}

In this large retrospective study, VRE colonization pressure was the most important risk factor for healthcare-associated VRE acquisition among subjects who were VRE-negative at the time of ICU admission. This effect could not be attributed to heterogeneity between individual ICUs, and was preserved even after adjusting for VRE importation pressure.

Here, VRE colonization pressure was defined to capture the burden of circulating VRE within a given ICU during a patient's ICU stay, whereas VRE importation pressure captured the VRE burden at the time of ICU admission. Previous studies in VRE acquisition have evaluated each of these factors independently. 6, 26,27 To our knowledge, this is the first study that has investigated the interaction between these two variables. The increased rates of VRE acquisition among patients who faced high VRE colonization pressure, regardless of importation pressure, may suggest that an important mechanism of VRE transmission is from known VRE-positive patients via healthcare workers or shared fomites as opposed to VRE transmitted from shared surfaces from prior patients. ${ }^{10,11}$ The types of risk factors examined may each suggest their own type of intervention: excess risk related to vancomycin may suggest the need for antibiotic stewardship; excess risk related to importation the need for environmental cleaning; and excess risk related to colonization pressure the need for surveillance and effective isolation.

In prior studies assessing the relationship between VRE colonization pressure and VRE acquisition, colonization pressure was defined as the daily mean point prevalence of VRE, calculated for each subject as the cumulative proportion of VRE-colonized ICU patients on each at-risk day. Bonten et al. studied the association between colonization pressure and VRE acquisition by collecting daily rectal cultures for all patients admitted to the medical ICU. ${ }^{6}$ They found that colonization pressure was the strongest predictor of VRE acquisition, even when considering other factors including antibiotic use. An effect related to ward antibiotic use was seen only when colonization pressure was low; interestingly, including vancomycin did not impact their results. In a subsequent study by Drees et al., VRE cultures were obtained from patients on ICU admission, twice weekly while the patient remained in the ICU, and at the time of ICU discharge. While colonization pressure was a significant predictor of VRE acquisition, prior VRE-positive room culture and prior room occupancy by VRE-colonized patients were also similarly important in their model. ${ }^{26}$

We utilized a definition of colonization pressure that deliberately placed weight on concurrently VRE-colonized patients who share a given ICU for many overlapping days. Similar definitions have been applied to acquisition of Clostridium difficile and MRSA, and under this definition $C$. difficile colonization pressure was an independent risk factor for $C$. difficile acquisition. ${ }^{16,19}$ When colonization pressure was defined as the proportion of all patient cultures positive for MRSA within 12 hours of a subject's admission, a reduction in colonization pressure was significantly associated with reductions in MRSA acquisition. Importation pressure has also been shown to be a strong predictor of regional variation in rates of Clostridium difficile infection. ${ }^{16}$ Colonization pressure and importation pressure may be synergistic in some scenarios, and the strength of a given association may depend on 
the organism studied, the prevention strategies employed, and other local institutional factors.

Here, healthcare-associated VRE acquisition was not significantly associated with factors related to prior bed occupants or neighbors. In contrast, previous studies have largely found that prior room occupancy by VRE-colonized patients does increase the risk of VRE acquisition. ${ }^{26-28}$ The strength of this association in these studies was modest, with low population attributable risk to VRE in the prior bed occupant. In one study, risk related to the prior bed occupant did not persist after the implementation of a more stringent room cleaning intervention. ${ }^{28}$ Interestingly, antibiotic use in the prior bed occupant was a risk factor for $C$. difficile infection at our own institution in a previous study but was not a risk factor for VRE acquisition in this study. ${ }^{17}$ This difference may speak to the greater hardiness of $C$. difficile compared to Enterococcus on ICU surfaces. Notably, nursing assignments in these ICUs were based on patient acuity rather than location, which may account for the lack of effect from neighbors. In addition, vancomycin use in the at-risk patient was a significant predictor of VRE acquisition in the univariate analysis but not in the final multivariable model, in contrast to prior studies that have demonstrated a relationship between vancomycin use and VRE infection in specific patient populations. ${ }^{29,} 30$ It is possible that vancomycin plays a role in increasing risk of VRE infection among patients colonized with VRE, although it may have less impact on VRE acquisition among VRE-negative patients.

This was a large study involving several units that took place over 5 years. We thoroughly evaluated the hospital milieu, incorporating numerous factors related to the at-risk patient, neighboring patients, and prior bed occupants. We also examined local VRE burden utilizing multiple definitions to best characterize circulating VRE. In addition, all patients in the study had an initial negative VRE culture, allowing us to clearly establish the temporal relationships between the exposure and the outcome. Nonetheless, this study also had limitations. The ICUs involved were heterogeneous. Several sensitivity analyses accounted for this, and the results were robust even within unique units. This was a single institution study, and these results may not be generalizable to other institutions with different VRE patterns. There may also have been rare cases in which VRE was falsely not detected, resulting in an underestimate of VRE acquisition. VRE screening during this study was performed using chromogenic agar. Although false negative or positive screening results cannot be completely excluded without confirmatory testing, prior studies show $99 \%$ sensitivity and specificity for chromogenic agar compared to alternative methods for VRE identification. ${ }^{31,32}$ We also did not collect environmental culture samples, which would have helped to directly characterize the circulating environmental VRE burden. Lastly, subsequent VRE swabs were not routinely collected for all patients, so this study likely underestimated the true rate of VRE acquisition. However, the relationships of interest did not change when we performed a restriction analysis that included only patients with a second VRE surveillance swab.

This study found that high VRE colonization pressure is a strong predictor of healthcareassociated VRE acquisition in the ICU. Other factors related to the local hospital milieu were less important in healthcare-associated VRE acquisition, including importation pressure and factors related to neighbors, prior bed occupants, and, somewhat surprisingly, 
to the local use of vancomycin. We found that the local environment can contribute to VRE colonization, which is likely to have important downstream consequences. Interventions seeking to reduce healthcare-associated VRE acquisition may wish to focus on ways to minimize transmission of VRE between patients with known VRE and the local hospital environment through effective isolation and other preventive measures.

\section{Supplementary Material}

Refer to Web version on PubMed Central for supplementary material.

\section{Acknowledgments}

Financial support: Dr. Freedberg was funded in part by NIH K23 DK111847 and by a Research Scholar Award from the American Gastroenterological Association.

Potential conflicts of interest: None.

\section{References}

1. Hidron AI, Edwards JR, Patel J, Horan TC, Sievert DM, Pollock DA, et al. National Healthcare Safety Network T and Participating National Healthcare Safety Network F. NHSN annual update: antimicrobial-resistant pathogens associated with healthcare-associated infections: annual summary of data reported to the National Healthcare Safety Network at the Centers for Disease Control and Prevention, 2006-2007. Infect Control Hosp Epidemiol. 2008; 29:996-1011. [PubMed: 18947320]

2. Salgado CD, Farr BM. Outcomes associated with vancomycin-resistant enterococci: a metaanalysis. Infect Control Hosp Epidemiol. 2003; 24:690-8. [PubMed: 14510253]

3. National Nosocomial Infections Surveillance S. National Nosocomial Infections Surveillance (NNIS) System Report, data summary from January 1992 through June 2004, issued October 2004. Am J Infect Control. 2004; 32:470-85. [PubMed: 15573054]

4. Song JY, Cheong HJ, Jo YM, Choi WS, Noh JY, Heo JY, et al. Vancomycin-resistant Enterococcus colonization before admission to the intensive care unit: a clinico-epidemiologic analysis. Am J Infect Control. 2009; 37:734-40. [PubMed: 19188004]

5. Kim TS, Kwon HL, Song SH, Song KH, Kim HB, Park KU, et al. Real-time PCR surveillance of vanA for vancomycin-resistant Enterococcus faecium. Mol Med Rep. 2012; 6:488-92. [PubMed: 22710424]

6. Bonten MJ, Slaughter S, Ambergen AW, Hayden MK, van Voorhis J, Nathan C, et al. The role of "colonization pressure" in the spread of vancomycin-resistant enterococci: an important infection control variable. Arch Intern Med. 1998; 158:1127-32. [PubMed: 9605785]

7. Amberpet R, Sistla S, Parija SC, Thabah MM. Screening for Intestinal Colonization with Vancomycin Resistant Enterococci and Associated Risk Factors among Patients Admitted to an Adult Intensive Care Unit of a Large Teaching Hospital. J Clin Diagn Res. 2016; 10:DC06-DC09.

8. Jung E, Byun S, Lee H, Moon SY, Lee H. Vancomycin-resistant Enterococcus colonization in the intensive care unit: clinical outcomes and attributable costs of hospitalization. American journal of infection control. 2014; 42:1062-6. [PubMed: 25278394]

9. Lankford MG, Collins S, Youngberg L, Rooney DM, Warren JR, Noskin GA. Assessment of materials commonly utilized in health care: implications for bacterial survival and transmission. Am J Infect Control. 2006; 34:258-63. [PubMed: 16765202]

10. Porwancher R, Sheth A, Remphrey S, Taylor E, Hinkle C, Zervos M. Epidemiological study of hospital-acquired infection with vancomycin-resistant Enterococcus faecium: possible transmission by an electronic ear-probe thermometer. Infect Control Hosp Epidemiol. 1997; 18:771-3. [PubMed: 9397374] 
11. Duckro AN, Blom DW, Lyle EA, Weinstein RA, Hayden MK. Transfer of vancomycin-resistant enterococci via health care worker hands. Arch Intern Med. 2005; 165:302-7. [PubMed: 15710793]

12. Lin MY, Hayden MK. Methicillin-resistant Staphylococcus aureus and vancomycin-resistant enterococcus: recognition and prevention in intensive care units. Crit Care Med. 2010; 38:S33544. [PubMed: 20647791]

13. Muto CA, Jernigan JA, Ostrowsky BE, Richet HM, Jarvis WR, Boyce JM, et al. SHEA guideline for preventing nosocomial transmission of multidrug-resistant strains of Staphylococcus aureus and enterococcus. Infect Control Hosp Epidemiol. 2003; 24:362-86. [PubMed: 12785411]

14. Recommendations for preventing the spread of vancomycin resistance. Recommendations of the Hospital Infection Control Practices Advisory Committee (HICPAC). MMWR Recomm Rep. 1995; 44:1-13.

15. Brown K, Valenta K, Fisman D, Simor A, Daneman N. Hospital ward antibiotic prescribing and the risks of Clostridium difficile infection. JAMA Intern Med. 2015; 175:626-33. [PubMed: 25705994]

16. Brown KA, Jones M, Daneman N, Adler FR, Stevens V, Nechodom KE, et al. Importation, Antibiotics, and Clostridium difficile Infection in Veteran Long-Term Care: A Multilevel CaseControl Study. Ann Intern Med. 2016; 164:787-94. [PubMed: 27088642]

17. Freedberg DE, Salmasian H, Cohen B, Abrams JA, Larson EL. Receipt of Antibiotics in Hospitalized Patients and Risk for Clostridium difficile Infection in Subsequent Patients Who Occupy the Same Bed. JAMA Intern Med. 2016; 176:1801-1808. [PubMed: 27723860]

18. Budding AE, Grasman ME, Eck A, Bogaards JA, Vandenbroucke-Grauls CM, van Bodegraven AA, et al. Rectal swabs for analysis of the intestinal microbiota. PLoS One. 2014; 9:e101344. [PubMed: 25020051]

19. Dubberke ER, Reske KA, Olsen MA, McMullen KM, Mayfield JL, McDonald LC, et al. Evaluation of Clostridium difficile-associated disease pressure as a risk factor for $\mathrm{C}$ difficileassociated disease. Arch Intern Med. 2007; 167:1092-7. [PubMed: 17533213]

20. Salmasian H, Freedberg DE, Friedman C. Deriving comorbidities from medical records using natural language processing. J Am Med Inform Assoc. 2013; 20:e239-42. [PubMed: 24177145]

21. Zimmerman JE, Kramer AA, McNair DS, Malila FM. Acute Physiology and Chronic Health Evaluation (APACHE) IV: hospital mortality assessment for today's critically ill patients. Crit Care Med. 2006; 34:1297-310. [PubMed: 16540951]

22. Singer M, Deutschman CS, Seymour CW, Shankar-Hari M, Annane D, Bauer M, et al. The Third International Consensus Definitions for Sepsis and Septic Shock (Sepsis-3). JAMA. 2016; 315:801-10. [PubMed: 26903338]

23. Brown KA, Khanafer N, Daneman N, Fisman DN. Meta-analysis of antibiotics and the risk of community-associated Clostridium difficile infection. Antimicrob Agents Chemother. 2013; 57:2326-32. [PubMed: 23478961]

24. Faleck DM, Salmasian H, Furuya EY, Larson EL, Abrams JA, Freedberg DE. Proton Pump Inhibitors Do Not Increase Risk for Clostridium difficile Infection in the Intensive Care Unit. Am J Gastroenterol. 2016; 111:1641-1648. [PubMed: 27575714]

25. Grambsch PM, Therneau TM. Proportional hazards tests and diagnostics based on weighted residuals. Biometrika. 1994; 81:515-526.

26. Drees M, Snydman DR, Schmid CH, Barefoot L, Hansjosten K, Vue PM, et al. Prior environmental contamination increases the risk of acquisition of vancomycin-resistant enterococci. Clin Infect Dis. 2008; 46:678-85. [PubMed: 18230044]

27. Huang SS, Datta R, Platt R. Risk of acquiring antibiotic-resistant bacteria from prior room occupants. Arch Intern Med. 2006; 166:1945-51. [PubMed: 17030826]

28. Datta R, Platt R, Yokoe DS, Huang SS. Environmental cleaning intervention and risk of acquiring multidrug-resistant organisms from prior room occupants. Arch Intern Med. 2011; 171:491-4. [PubMed: 21444840]

29. Heisel RW, Sutton RR, Mascara GP, Winger DG, Weber DR, Lim SH, et al. Vancomycin-resistant enterococci in acute myeloid leukemia and myelodysplastic syndrome patients undergoing 
induction chemotherapy with idarubicin and cytarabine. Leuk Lymphoma. 2017; 58:2565-2572. [PubMed: 28351179]

30. Nerandzic MM, Mullane K, Miller MA, Babakhani F, Donskey CJ. Reduced acquisition and overgrowth of vancomycin-resistant enterococci and Candida species in patients treated with fidaxomicin versus vancomycin for Clostridium difficile infection. Clin Infect Dis. 2012; 55(Suppl 2):S121-6. [PubMed: 22752860]

31. Peterson JF, Doern CD, Kallstrom G, Riebe KM, Sander T, Dunne WM Jr, et al. Evaluation of Spectra VRE, a new chromogenic agar medium designed to screen for vancomycin-resistant Enterococcus faecalis and Enterococcus faecium. J Clin Microbiol. 2010; 48:4627-9. [PubMed: 20943872]

32. Anderson NW, Buchan BW, Young CL, Newton DW, Brenke C, Lapsley L, et al. Multicenter clinical evaluation of VRESelect agar for identification of vancomycin-resistant Enterococcus faecalis and Enterococcus faecium. J Clin Microbiol. 2013; 51:2758-60. [PubMed: 23761153] 


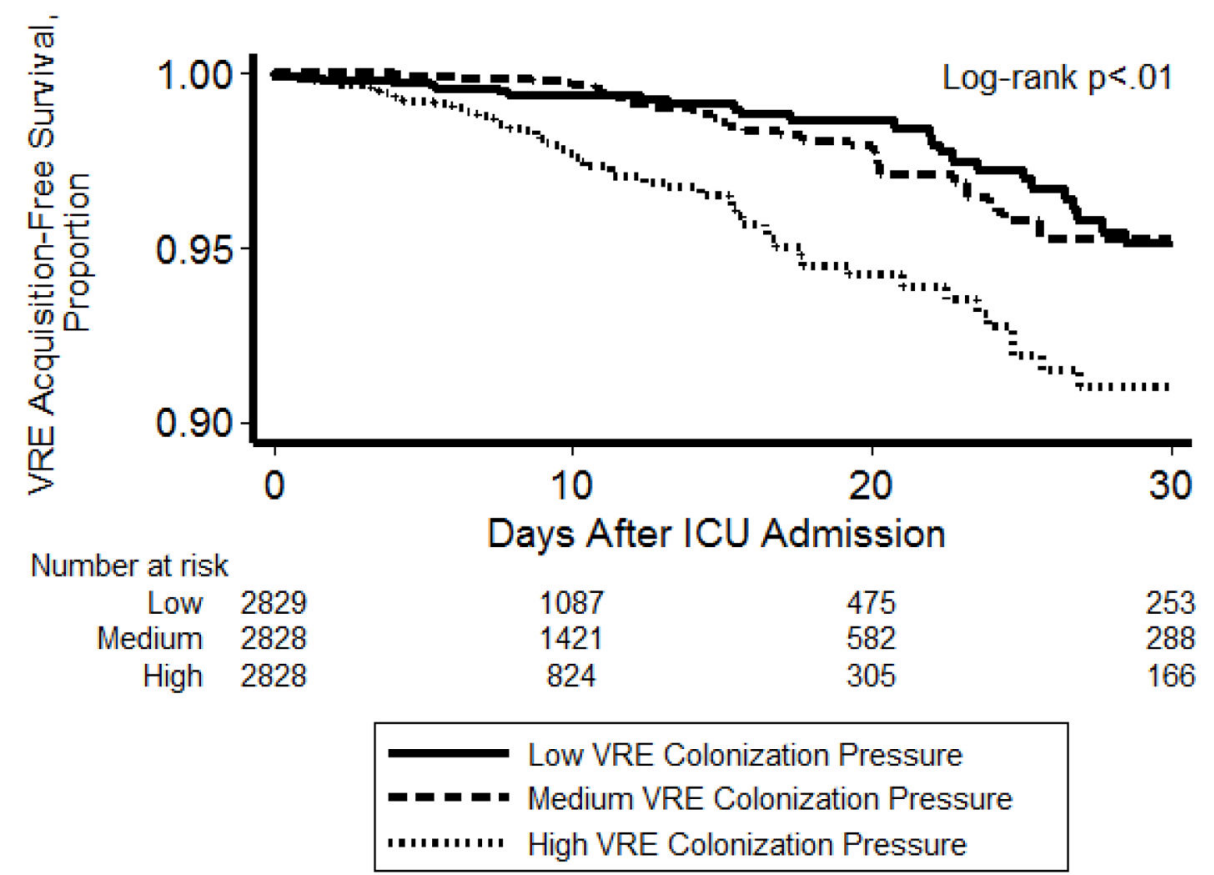

Figure 1. Vancomycin-resistant enterococci (VRE) acquisition-free survival, stratified by VRE colonization pressure

Patients who had negative VRE (vancomycin-resistant Enterococcus) rectal swabs at the time of ICU (intensive care unit) admission were followed until VRE acquisition, death, or for up to 30 days. VRE colonization pressure was organized into tertiles and was calculated for each patient as the number of VRE-positive patient-days on that patient's unit, divided by the patient's at-risk time (see manuscript for additional details). 
Table I

Patient characteristics, stratified by acquisition of vancomycin-resistant enterococci (VRE)

\begin{tabular}{|c|c|c|c|c|}
\hline Characteristics & VRE acquisition $(n=161)$ & No VRE acquisition $(\mathrm{n}=\mathbf{8 , 3 2 4})$ & Total $(n=8,485)$ & p-value \\
\hline \multicolumn{5}{|c|}{ Baseline demographics and comorbidities } \\
\hline Male sex & $91(57 \%)$ & $4,384(53 \%)$ & $4,475(53 \%)$ & 0.33 \\
\hline \multicolumn{5}{|l|}{ Age } \\
\hline $18-49$ years & $63(39 \%)$ & $2,766(33 \%)$ & $2,829(33 \%)$ & 0.06 \\
\hline $50-70$ years & $58(36 \%)$ & $2,770(33 \%)$ & $2,828(33 \%)$ & \\
\hline$>70$ years & $40(25 \%)$ & $2,788(33 \%)$ & $2,828(33 \%)$ & \\
\hline \multicolumn{5}{|l|}{ Race } \\
\hline Black & $16(10 \%)$ & $848(10 \%)$ & $864(10 \%)$ & 0.93 \\
\hline Hispanic & $41(25 \%)$ & $2,293(28 \%)$ & $2,334(28 \%)$ & \\
\hline White & $51(32 \%)$ & $2,601(31 \%)$ & $2,652(31 \%)$ & \\
\hline Other/unknown & $53(33 \%)$ & $2,582(31 \%)$ & $2,635(31 \%)$ & \\
\hline \multicolumn{5}{|l|}{ Season of admission ${ }^{*}$} \\
\hline Winter & $44(27 \%)$ & $2,148(26 \%)$ & $2,192(26 \%)$ & 0.78 \\
\hline Spring & $40(25 \%)$ & $1,978(24 \%)$ & $2,018(24 \%)$ & \\
\hline Summer & $41(25 \%)$ & $2,035(24 \%)$ & $2,076(24 \%)$ & \\
\hline Autumn & $36(22 \%)$ & $2,163(26 \%)$ & $2,199(26 \%)$ & \\
\hline \multicolumn{5}{|l|}{ ICU location } \\
\hline 1 & $11(7 \%)$ & $1,989(24 \%)$ & $2,000(24 \%)$ & $<0.01$ \\
\hline 2 & $2(1 \%)$ & $84(1 \%)$ & $86(1 \%)$ & \\
\hline 3 & $42(26 \%)$ & $3,020(36 \%)$ & $3,062(36 \%)$ & \\
\hline 4 & $1(0.6 \%)$ & $31(0.4 \%)$ & $32(0.4 \%)$ & \\
\hline 5 & $50(31 \%)$ & $1,704(20 \%)$ & $1,754(21 \%)$ & \\
\hline 6 & $55(34 \%)$ & $1,496(18 \%)$ & $1,551(18 \%)$ & \\
\hline \multicolumn{5}{|l|}{ Charlson comorbidity index } \\
\hline $0-1$ & $43(27 \%)$ & $2,840(34 \%)$ & $2,883(34 \%)$ & 0.06 \\
\hline $1-2$ & $49(30 \%)$ & $2,597(31 \%)$ & $2,646(31 \%)$ & \\
\hline 23 & $69(43 \%)$ & $2,887(35 \%)$ & $2,956(35 \%)$ & \\
\hline \multicolumn{5}{|l|}{ Laboratory values at ICU admission } \\
\hline Sodium $<135$ or $>145 \mathrm{mEq} / \mathrm{L}$ & $46(29 \%)$ & $2,210(27 \%)$ & $2,256(27 \%)$ & 0.57 \\
\hline Creatinine $>2 \mathrm{mg} / \mathrm{dL}$ & $54(34 \%)$ & $1,555(19 \%)$ & $1,609(19 \%)$ & $<0.01$ \\
\hline Albumin $<2.5 \mathrm{~g} / \mathrm{dL}$ & $54(34 \%)$ & $1,736(21 \%)$ & $1,790(21 \%)$ & $<0.01$ \\
\hline Hematocrit $<35 \%$ & $23(14 \%)$ & $991(12 \%)$ & $1,014(12 \%)$ & 0.36 \\
\hline White blood cells $<4$ or $>12 \times 10^{9} / \mathrm{L}$ & $77(48 \%)$ & $3,373(41 \%)$ & $3,450(41 \%)$ & 0.06 \\
\hline Sodium $<135$ or $>145 \mathrm{mEq} / \mathrm{L}$ & $46(29 \%)$ & $2,210(27 \%)$ & $2,256(27 \%)$ & 0.57 \\
\hline \multicolumn{5}{|l|}{ Treatment received in ICU } \\
\hline Antibiotics & $126(78 \%)$ & $5,964(72 \%)$ & $6,090(72 \%)$ & 0.07 \\
\hline
\end{tabular}

$J$ Hosp Infect. Author manuscript; available in PMC 2020 January 01. 


\begin{tabular}{lcccc}
\hline Characteristics & VRE acquisition $(\mathbf{n}=\mathbf{1 6 1})$ & No VRE acquisition $(\mathbf{n}=\mathbf{8 , 3 2 4})$ & Total $(\mathbf{n}=\mathbf{8 , 4 8 5})$ & p-value \\
\hline Immunosuppressants & $102(63 \%)$ & $3,356(40 \%)$ & $3,458(41 \%)$ & $<0.01$ \\
Proton pump inhibitors & $90(56 \%)$ & $4,071(49 \%)$ & $4,161(49 \%)$ & 0.08 \\
Recent surgery & $26(16 \%)$ & $2,063(25 \%)$ & $2,089(25 \%)$ & 0.01 \\
Mechanical ventilation & $112(70 \%)$ & $4,264(51 \%)$ & $4,376(52 \%)$ & $<0.01$ \\
Dialysis & $30(19 \%)$ & $591(7 \%)$ & $621(7 \%)$ & $<0.01$ \\
\hline
\end{tabular}

* Season of admission was defined as follows: winter, December-February; spring, March-May; summer, June-August; autumn, SeptemberNovember. ICU: intensive care unit. 


\section{Table II}

Local hospital milieu factors, stratified by acquisition of vancomycin-resistant enterococci (VRE)

\begin{tabular}{|c|c|c|c|c|}
\hline Characteristics & VRE acquisition $(n=161)$ & No VRE acquisition $(n=8,324)$ & Total $(n=8,485)$ & p-value \\
\hline \multicolumn{5}{|l|}{ At-risk patient } \\
\hline Vancomycin use & $132(82 \%)$ & $4,721(57 \%)$ & $4,853(57 \%)$ & $<0.01$ \\
\hline \multicolumn{5}{|c|}{ Prior bed occupant } \\
\hline VRE colonization & $69(43 \%)$ & $3,487(42 \%)$ & $3,556(42 \%)$ & 0.81 \\
\hline Vancomycin use & $75(47 \%)$ & $3,331(40 \%)$ & $3,406(40 \%)$ & 0.09 \\
\hline \multicolumn{5}{|c|}{ Neighboring patient(s) } \\
\hline VRE colonization & $35(22 \%)$ & $1,497(18 \%)$ & $1,532(18 \%)$ & 0.22 \\
\hline Vancomycin use & $64(40 \%)$ & $2,677(32 \%)$ & $2,741(32 \%)$ & 0.04 \\
\hline \multicolumn{5}{|l|}{ Unit-level } \\
\hline \multicolumn{5}{|c|}{ VRE colonization pressure } \\
\hline Low & $33(21 \%)$ & $2,836(34 \%)$ & $2,869(34 \%)$ & $<0.01$ \\
\hline Medium & $61(38 \%)$ & $2,806(34 \%)$ & $2,867(34 \%)$ & \\
\hline High & $67(42 \%)$ & $2,682(32 \%)$ & $2,749(32 \%)$ & \\
\hline \multicolumn{5}{|c|}{ VRE importation pressure } \\
\hline Low & $42(26 \%)$ & $2,787(34 \%)$ & $2,829(33 \%)$ & 0.01 \\
\hline Medium & $48(30 \%)$ & $2,780(33 \%)$ & $2,828(33 \%)$ & \\
\hline High & $71(44 \%)$ & $2,757(33 \%)$ & $2,828(33 \%)$ & \\
\hline \multicolumn{5}{|l|}{ Vancomycin use } \\
\hline Low & $59(37 \%)$ & $2,781(33 \%)$ & $2,840(33 \%)$ & 0.55 \\
\hline Medium & $48(30 \%)$ & $2,799(34 \%)$ & $2,847(34 \%)$ & \\
\hline High & $54(33 \%)$ & $2,744(33 \%)$ & $2,798(33 \%)$ & \\
\hline
\end{tabular}

$J$ Hosp Infect. Author manuscript; available in PMC 2020 January 01. 
Table III

Multivariable model of risk factors for vancomycin-resistant enterococci (VRE) acquisition

\begin{tabular}{lc}
\hline Risk factor & VRE acquisition Adjusted hazard ratio $(95 \%$ CI) \\
\hline VRE colonization pressure & Reference \\
Low & $0.82(0.54-1.26)$ \\
Medium & $1.79(1.19-2.70)$ \\
High & \\
ICU location & $0.40(0.21-0.79)$ \\
1 & $0.62(0.15-2.60)$ \\
2 & $0.71(0.47-1.09)$ \\
3 & $0.94(0.13-7.00)$ \\
4 & Reference \\
5 & $1.18(0.80-1.73)$ \\
6 & \\
Charlson comorbidity index & Reference \\
$0-1$ & $1.12(0.74-1.69)$ \\
$1-2$ & $1.27(0.86-1.88)$ \\
$\geq 3$ & $1.52(1.09-2.13)$ \\
Creatinine $>2$ mg/dL &
\end{tabular}

